\title{
Large Pelvic Arteriovenous Malformation - A Bloody Near Miss
}

\author{
Yun Le Linn ${ }^{1}$, Kiang Hiong Tay ${ }^{2}$, and Tze Tec Chong ${ }^{1}$ \\ ${ }^{1}$ Department of Vascular Surgery and ${ }^{2}$ Division of Radiological Sciences, Singapore General Hospital, Singapore
}

Our patient, a healthy 32-year-old man, was referred for non-specific left flank pain with no associated symptoms. Physical examination findings were unremarkable. Computed tomography (CT) urography revealed a circumscribed enhancing mass in the left hemipelvis initially thought to be obturator lymphadenopathy from possible lymphoma (Fig. 1). A CT-guided biopsy yielded pulsatile blood. CT angiography fortunately did not demonstrate any extravasation, but it did reveal an arteriovenous malformation (AVM) with a large venous aneurysm mimicking a pelvic mass. This was further characterized on catheter angiography, which demonstrated multiple feeding vessels from the inferior mesenteric artery (IMA) and left internal iliac artery (IIA) with involvement of the rectal wall (Fig. 2). Our patient underwent staged angioembolizations over two sittings, beginning with $2.65 \mathrm{~mL}$ of Onyx18 (Medtronic, Dublin, Ireland) delivered to four IIA branches using a right common femoral artery (CFA) crossover approach and subsequently to IMA branches using a left retrograde CFA approach (Fig. 3). He achieved symptom resolution with a small residual AVM component on magnetic resonance angiography (Fig. 4).

Our patient's AVM was unique in that it received a blood supply from multiple vessels from the left IIA and IMA. Moreover, it contributed to the rectum's vascular supply, complicating treatment due to the potential for rectal ischemia. Management options include surveillance with serial imaging, endovascular techniques such as coiling or glue embolization to treat the nidus, or open surgical resection $[1,2]$. Given the unique profile of our patient's AVM, staged embolization of the feeding vessels to achieve symptom relief was preferred starting with the left IIA branches and progressing to the IMA branches if symptoms persist. This case highlights that AVMs can be difficult to diagnose on
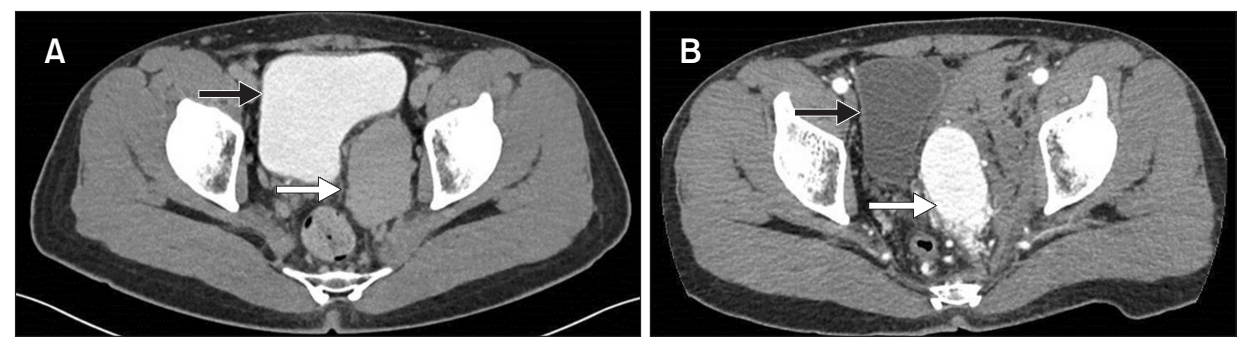

Fig. 1. (A) Circumscribed left hemipelvis-enhancing mass was detected on the venous phase of computed tomography (CT) urography. (B) Corresponding image on CT angiography indicated that the left hemipelvic mass was actually a venous aneurysm of a pelvic arteriovenous malformation (AVM). A hematoma resulted from the biopsy, but no active contrast extravasation was seen. The black arrow indicates the bladder, while the white arrow indicates the AVM.

Received July 9, 2021, Revised September 1, 2021, Accepted September 9, 2021, Published online September 28, 2021

Corresponding author: Yun Le Linn, Department of Vascular Surgery, Singapore General Hospital, Level 5; Academia, 20 College Road, Singapore 169856, Singapore

Tel: 65-62207765, Fax: 65-62223322, Email: yunle.linn@mohh.com.sg, https://orcid.org/0000-0002-7119-4178

Copyright (C) 2021 The Korean Society for Vascular Surgery

This is an Open Access article distributed under the terms of the Creative Commons Attribution Non-Commercial License (http://creativecommons.org/licenses/by-nc/4.0) which permits unrestricted non-commercial use, distribution, and reproduction in any medium, provided the original work is properly cited.

Cite this article; Vasc Specialist Int 2021. https://doi.org/10.5758/vsi.210050 

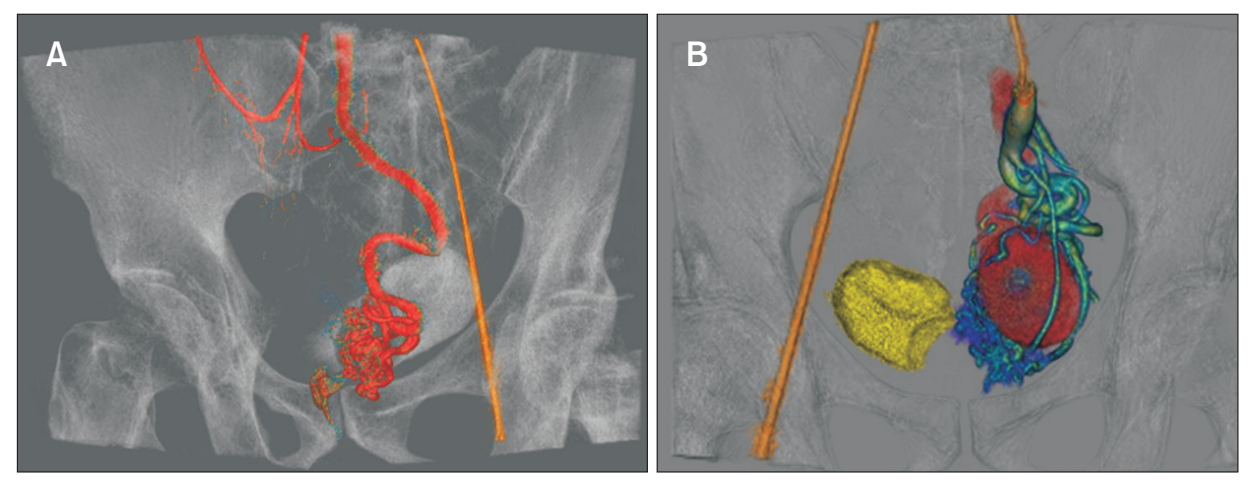

Fig. 2. Catheter angiography images demonstrated the feeding vessels from branches of the inferior mesenteric artery $(A)$ and internal iliac artery (B).

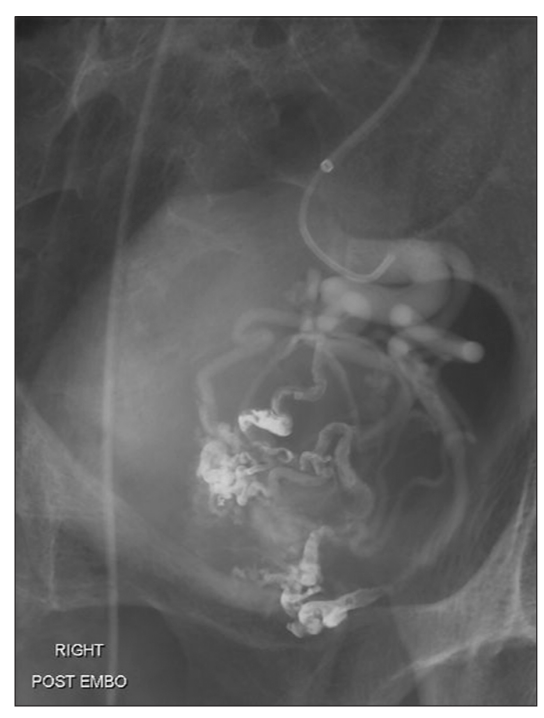

Fig. 3. Completion angiography after embolization of the feeding vessels.

CT urography, in which images are acquired in the noncontrasted and venous phases only and that contrastenhanced CT should be performed prior to biopsy to avoid

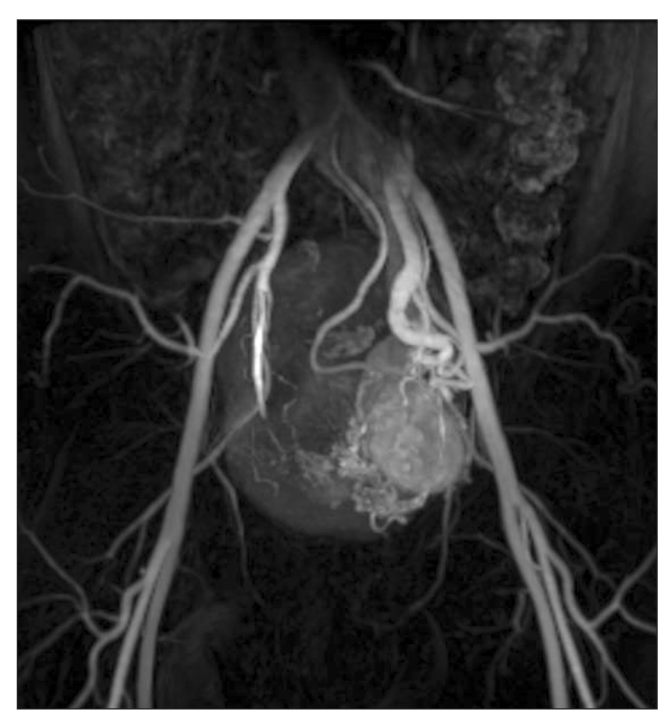

Fig. 4. Magnetic resonance angiography showed a small residual arteriovenous malformation component and symptom resolution.

catastrophic bleeding. Thankfully, our patient's bleeding was self-limiting and he did not require transfusion.

\section{REFERENCES}

1) Coulier B, De Wispelaere JF, Bueres I, Pierard F, Deprez FC, Maldague P, et al. Acute massive congestive ischaemic colitis related to inferior mesenteric arteriovenous malformation. BJR Case Rep 2016;2:20150275.

2) Ashley RA, Patterson DE, Bower TC, Stanson AW. Large congenital pelvic arteriovenous malformation and management options. Urology 2006;68:203.e11-3. 